\title{
High Energy Spin Waves in Iron CONF-910903--9 \\ Measured by Neutron Scattering DE91 017868
}

\author{
A.T. Boothroyd", T.G. Perring*, A.D. Taylor ${ }^{\dagger}$, \\ D.McK Paul ${ }^{\#}$ and $\mathrm{H}_{\lambda}{ }^{\mathrm{A}}$ Mook
}

\#Physics Department, University of Warwick, Coventry, CV4 7AL, UK

*Clarendon Laboratory, Parks Road, Oxford, OX1 3PU, UK

†Rutherford Appleton Laboratory, Chilton, Didcot, OX11 OQX, UK

¥Oak Ridge National Laboratory, Oảk Ridge, Tennessee 37831, USA

\begin{abstract}
We present new results for the spin wave dispersion relation

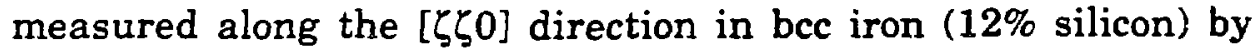
time-of-flight, neutron inelastic scattering. The excitations were followed to the zone boundary, where they are spread over a range of energies around $300 \mathrm{meV}$.
\end{abstract}

Keywords: Ferromagnetism, Itinerant electrons - Spin Waves, Magnetic Excitations, Neutron Scattering - Inelastic

Running Title: Spin waves in iron

-The submitted manuscript has been authored by a contractor of the U.S. Government under contract No. DE. AC05-840R21400. Accordingly, the U.S. Govemment retains a nonerclusive. royalty-free license to publish or reproduce the published form of this contribution, or nllow others to do so, for U.S. Government purposes.

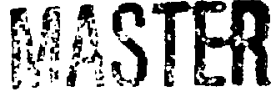
DISTRIBUTION OF THIS DOCUMENT IS UNLAMITED

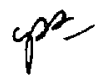

$$
\text { Ti be piejeitid cut }
$$

1

$$
\text { ICM91, Edinburgh }
$$


The spin wave modes in iron display some interesting and unusual features which cannc be explained in terms of a localised moment ferromagnet. Neutron scattering experiments have revealed an excitation spectrum which consists of sharp, isotropic spin waves below $100 \mathrm{meV}[1,2]$, but which at higher energies is observed to broaden, decrease in intensity and become anisotropic in wavevector [3].

These effects have been interpreted as arising from a degree of itineracy amongst the $3 d$ electrons, and have led to the development of a method to obtain the magnetic properties of the ferromagnetic $3 d$ metals from spin-polarised band calculations [4]. The theory has been compared with experiment for excitations up

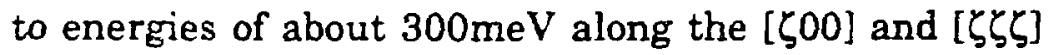
symmetry directions in iron, and satisfactory agreement has been achieved with the model parameters used. The excitation spectrum along the

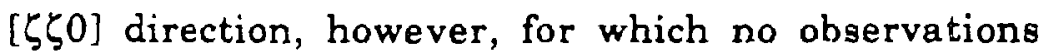
were previously available above $100 \mathrm{meV}$, was found to be highly sensitive to these parameters [5].

We have undertaken a series of neutron scattering measurements on iron at the spallation neutron source ISIS with the object of extending the energy scale of the experimental data to above $300 \mathrm{meV}$, and of obtaining information on the high energy part of the excitation spectrum along the $[\zeta \zeta 0]$ symmetry direction. This paper is a preliminary account of the 
iesults. We reported earlier [6] on our measurements along the [ร00] direction.

The experimental approach was very similar to that described previously [6], with the exception that the data were taken on the MARI spectrometer instead of on HET. Briefly, the iron crystal is made from the isotope ${ }^{54} \mathrm{Fe}$ alloyed with about $12 \%$ silicon to stabilise the bcc phase, and has a total mass of $170 \mathrm{~g}$. The silicon is known to decrease the spin wave stiffness by about $15 \%$, but otherwise the magnetic properties are expected to be similar to those of elemental iron. Like HET, MARI is a direct geometry, time-of-flight spectrometer which uses a rotating Fermi chopper phased to the source proton pulse to select the incident neutron energy. The resolution in energy transfer is approximately $2 \%$ of the incident energy. Unlike HET, however, MARI has all its detectors situated $4 \mathrm{~m}$ from the sample, and has continuous coverage with virtually constant energy resolution for scattering angles in the range $3^{\circ}$ to $135^{\circ}$.

The crystal was aligned with the (200) and (020) reflections in the scattering plane, and spin waves were measured at room temperature about the reciprocal lattice vector (200). In the time-of-flight method the incident neutron energy and scattering angle are fixed, so the wavevector, $\mathbf{Q}$, and energy transfer, $\omega$, vary according to the arrival time, $t$, of the neutrons at the detector. To measure spin waves the crystal was aligned so that the locus of $\mathbf{Q}(t)$ in one 
detector passed through the reciprocal lattice point (200) along the $[\zeta \zeta 0]$ direction. Spin waves appear as peaks in intensity whenever the time-of-flight trajectory intersects the dispersion curve.

Figure 1 shows the observed intensity transformed from time-of-flight to energy transfer for an incident energy of $295 \mathrm{meV}$. The scattering has been corrected for the energy dependence of the detector efficiency and for the $\mathrm{k}_{\mathrm{f}} / \mathrm{k}_{\mathrm{i}}$ phase space term in the cross-section, but not for the magnetic form factor. Two spin waves can be resolved on either side of (200) at energies of $120 \mathrm{meV}$ and $180 \mathrm{meV}$, and a third mode is also visible at $210 \mathrm{meV}$ in the adjacent zone. Also shown in figure 1 is an estimate of the multiple scattering and multiphonon background obtained with an appropriate solid angle correction from a set of detectors at sufficiently high scattering angle so as to contain no magnetic scattering. We note that on MARI the assessment of non-magnetic scattering is easier than on HET because all the detectors have the same energy resolution.

To obtain the peak energies and widths we subtracted the background scattering from the signal, corrected for the magnetic form factor and fitted the peaks with Gaussian functions, allowing the centres, widths and intensities to vary.

In figure 2 we present the spin wave dispersion curve obtained in this way for the $[\zeta \zeta 0]$ direction, with the 
bars indicating the trajectories of the time-of-flight scans between the half-maxima of the peaks. No attempt has been made yet to allow for the effects of resolution, so some details will need to be revised in the future. Also, it should be noted that figure 2 contains no information on the intensity of the scattering, so the excitations around the zone boundary may appear somewhat exaggerated. Nevertheless, the dispersion curve is seen to increase monotonically to the zone boundary, at which point the excitations extend in energy from about $200 \mathrm{meV}$ to at least $350 \mathrm{meV}$. Qualitatively the excitations resemble

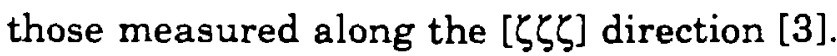

The present results, together with those from our previous experiment on ISIS [6], complete the missing high energy parts of the spin wave dispersion curve of iron along the major symmetry directions. Once a detailed analysis of the data has been performed to account for resolution effects we aim to compare the spectrum of excitations with the form of the generalised susceptibility, $\chi(\mathbf{Q}, \omega)$, predicted from the band theory calculation. This new information at

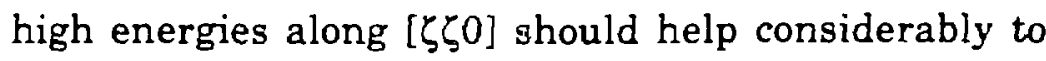
fix the parameters of the model and to make more reliable predictions of $\chi(Q, \omega)$ possible throughout the entire Brillouin zone. 


\title{
References
}

[1] M.F. Collins, V.J. Minkiewicz, R. Nathans, L. Passell and

G. Shirane, Phys. Rev. 179 (1969) 417

[2] H.A. Mook and R.M. Nicklow, Phys. Rev. B7 (1973) 336

[3] D.McK. Paul, P.W. Mitchell, H.A. Mook, and U.

Steigenberger, Phys. Rev. B38 (1988) 580

[4] J.A. Blackman, T. Morgan and J.F. Cooke, Phys. Rev.

Lett. 55 (1985) 2814

[5] J.A. Blackman, Private Communication

[6] T.G. Perring, A.T. Boothroyd, D.Mck Paul, A.D. Taylor,

R. Osborn, R.J. Newport, J.A. Blackman and H.A. Mook, J.

Appl. Phys. 69 (1991) 6219

\section{DISCLAIMER}

\begin{abstract}
This report was prepared as an account of work sponsored by an agency of the United States Government. Neither the United States Government nor any agency thereof, nor any of :heir employees, makes any warranty, express or implied, or assumes any legal liability or responsibility for the accuracy, completeness, or usefulness of any information, apparatus, product, or process disclosed, or represents that its use would not infringe privately owned rights. Reference herein to any specific commercial product, process, or service by trade name, trademark, manufacturer, or otherwise does not necessarily constitute or imply its endorsement, recom. mendation, or favoring by the United States Government or any agency thereof. The views and opinions of authors expressed herein do not necessarily state or reflect those of the United States Government or any agency thereof.
\end{abstract}


Figure Captions

Figure 1. Spin wave scattering from $\mathrm{Fe}(12 \% \mathrm{Si})$ measured on MARI with an incident energy of $295 \mathrm{meV}$. The smooth curve is an estimate of the multiple scattering and multiphonon background. The upper scale indicates the reduced scattering

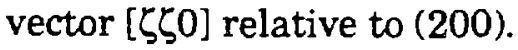

Figure 2. Spin wave dispersion curve of $\mathrm{Fe}(12 \% \mathrm{Si})$ measured

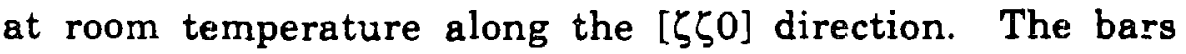
represent the time-of-flight scans and correspond to the full width at half maximum of the peaks, and the vertical broken line indicates the zone boundary (Z.B.). 
Intensity (arb. units)

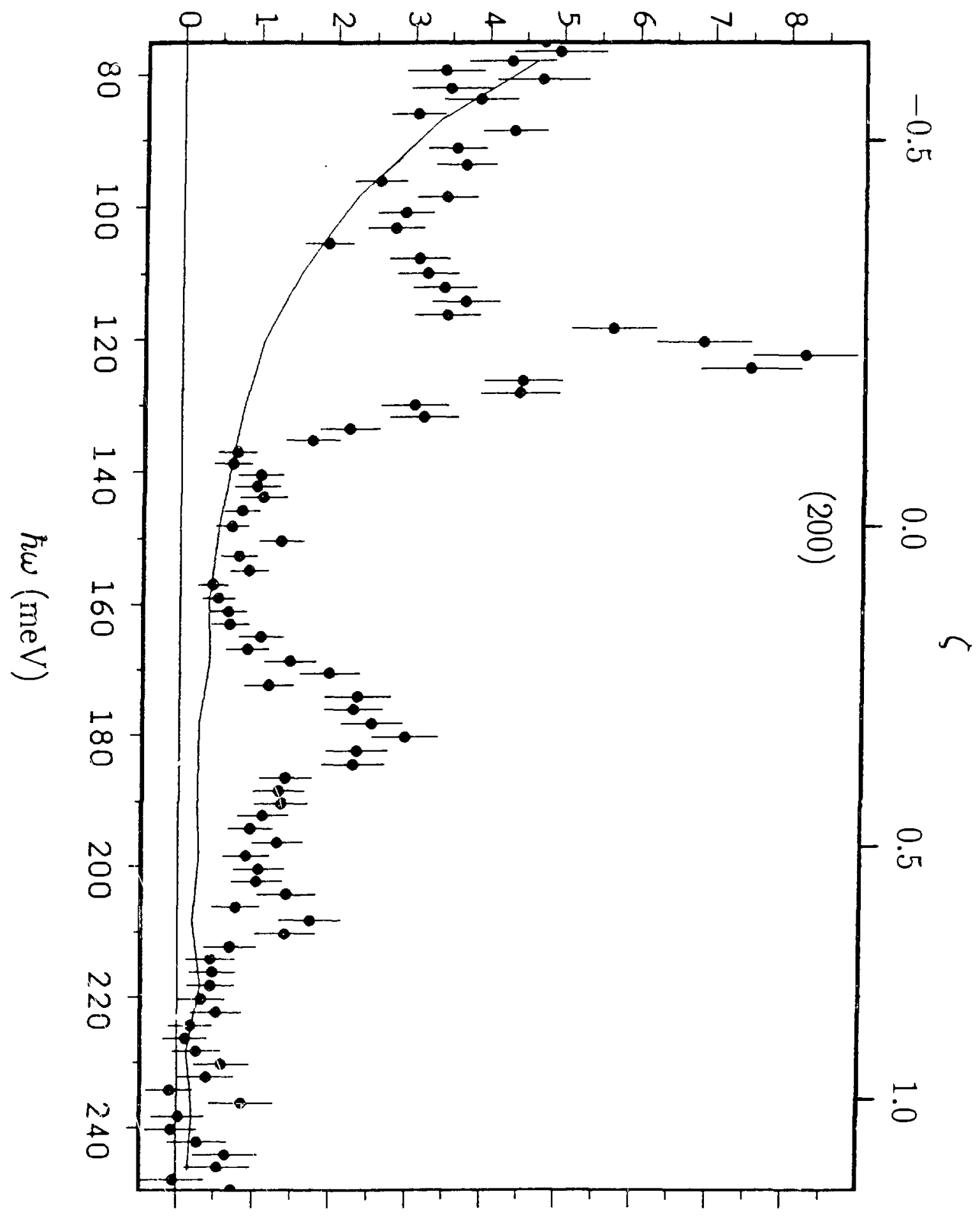


